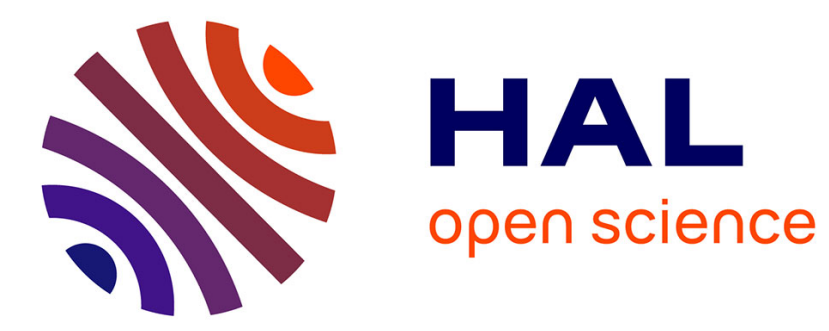

\title{
Spin glasses and roughening transition
}

\author{
G. Toulouse, J. Vannimenus, J.M. Maillard
}

\section{To cite this version:}

G. Toulouse, J. Vannimenus, J.M. Maillard. Spin glasses and roughening transition. Journal de Physique Lettres, 1977, 38 (22), pp.459-461. 10.1051/jphyslet:019770038022045900 . jpa-00231420

\section{HAL Id: jpa-00231420 https://hal.science/jpa-00231420}

Submitted on 1 Jan 1977

HAL is a multi-disciplinary open access archive for the deposit and dissemination of scientific research documents, whether they are published or not. The documents may come from teaching and research institutions in France or abroad, or from public or private research centers.
L'archive ouverte pluridisciplinaire HAL, est destinée au dépôt et à la diffusion de documents scientifiques de niveau recherche, publiés ou non, émanant des établissements d'enseignement et de recherche français ou étrangers, des laboratoires publics ou privés. 


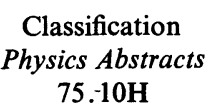

\title{
SPIN GLASSES AND ROUGHENING TRANSITION
}

\author{
G. TOULOUSE, J. VANNIMENUS and J. M. MAILLARD \\ Laboratoire de Physique de l'Ecole Normale Supérieure, \\ 24, rue Lhomond, 75231 Paris Cedex 05, France
}

(Reçu le 16 septembre 1977, accepté le 19 octobre 1977)

\begin{abstract}
Résumé. - Nous suggérons l'existence d'une connexion entre deux problèmes jusqu'ici séparés : la transition rugueuse, étudiée en théorie de la croissance cristalline, et la transition verre de spin, qui apparaît dans des matériaux magnétiques désordonnés.
\end{abstract}

\footnotetext{
Abstract. - We suggest the existence of a connection between two hitherto unrelated problems : the roughening transition, studied in crystal growth theory, and the spin glass transition, which occurs in disordered magnets.
}

We suggest a connection between the roughening transition [1] and the spin glass transition [2].

This connection is best described on the upper part of figure 1. That is a schematic phase diagram for an Ising magnet; on the vertical axis is plotted the temperature; on the horizontal axis is plotted some sort of impurity concentration (this is made more precise below, within the frustration model), a measure of disorder, so that $x=0$ corresponds to the pure Ising magnet. It is now widely accepted that besides the paramagnetic $(P)$ and ferromagnetic $(F)$ phases, a spin glass (S.G.) phase may exist, as shown on the figure. Now, for the pure Ising magnet (vertical axis on the figure), besides the usual Curie transition temperature $T_{\mathrm{c}}$, another transition temperature $T_{\mathrm{R}}$ is indicated : this is the roughening transition, which has been much studied during the last thirty years by crystal growth experts, and which corresponds to the roughening of wall defects. Below $T_{\mathrm{R}}$, a wall defect has a finite width ; this width diverges at $T_{R}$, so that, above $T_{R}$, the wall is rough, instead of being smooth.

We suggest that the spin glass transition (between spin glass and paramagnetic phases) is the continuation of the roughening transition, when the disorder is increased. This implies that the roughening transition temperature $T_{\mathrm{R}}$ (dotted line on the figure) may decrease less rapidly than $T_{\mathrm{c}}$, and that $T_{\mathrm{R}}$ may even cross $T_{\mathrm{c}}$, rather than vanish with $T_{\mathrm{c}}$.

In dimension $2, T_{\mathrm{R}}$ is known [3] to be reduced to zero and the spin glass transition is also presumably [4] brought down to the horizontal axis, as shown on the lower part of figure 1. If our suggestion is correct, the whole transition line (roughening and spin glass) would rise, for Ising systems, when the space dimensionality is raised above dimension 2 .
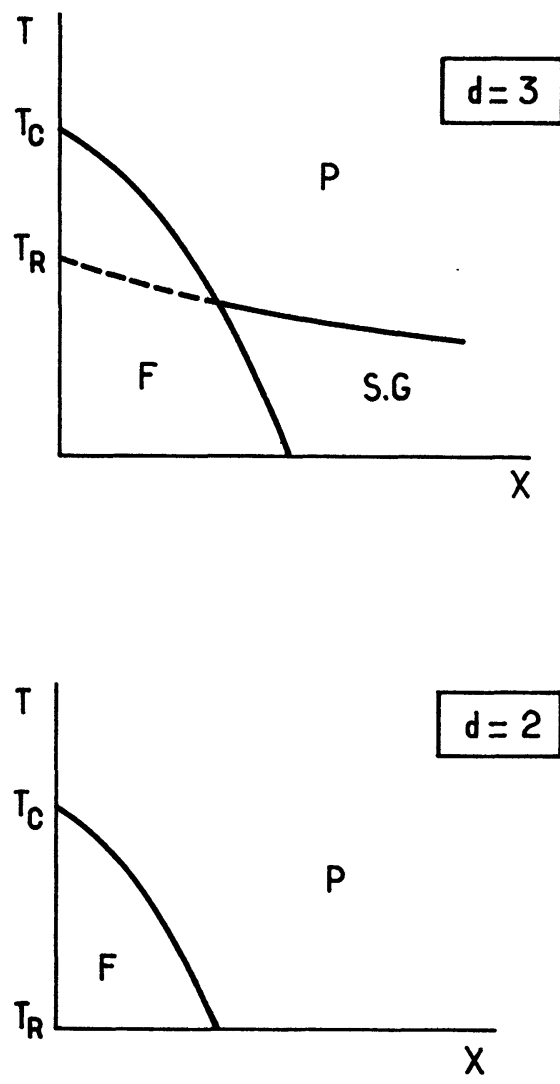

Fig. 1. - Proposed schematic phase diagram of an Ising magnet, showing the ferromagnetic (F), paramagnetic (P) and spin-glass (S.G.) phases, as a function of frustration disorder (e.g., $x=$ concentration of antiferromagnetic bonds). $T_{\mathrm{R}}$ is the roughening transition temperature, above which the width of a domain wall diverges : as the disorder increases, we conjecture that $T_{\mathrm{R}}$ decreases more slowly than the Curie temperature $T_{\mathrm{c}}$ and that the roughening transition is continued by the spin glass-paramagnetic transition $T_{\text {S.G. }}$ In dimension $d=2, T_{\mathrm{R}}$ is reduced to zero, as well as $T_{\text {s.G. }}$. 
After this presentation of the content of our conjecture, we discuss some plausibility arguments, explain how we were led to them and finally establish some perspectives, which may stimulate further work.

A spin glass phase has an unusual type of order. There is no long range magnetic order, which clearly distinguishes it from a ferromagnetic phase. However, the distinction from the paramagnetic phase is subtle and it is a present-day goal to obtain a clear picture of the spin glass transition, that is from the spin glass phase to the paramagnetic phase.

A spin glass system has a large number of ground states into which it may condense. This is best understood within the frustration picture which has recently been developed [5]. The system contains a frustration network (frustrated plaquettes in dimension 2, frustrated lines in dimension $3, \ldots$ ) which acts as an obligatory source or sink of defects, so that there exist zero-point intrinsic defects. A ground state is characterized by a configuration of these defects bounded by the frustration network; in dimension 2 , for instance, this means a pairing of frustrated plaquettes by string defects ; in dimension 3 , this means a setting of wall defects on a linear frustration network, which can be imagined as the result of the dipping of a wire armature in a soapy solution. There are many ways of realizing such a pairing or such a setting, and this correspond's to the multiplicity of ground states. One may go from one ground state to another by a crossover between defects. For instance, in dimension 2 , instead of pairing plaquettes 1 and 2 , and plaquettes 3 and 4 , we could have a pairing of plaquettes 1 and 3 , and of plaquettes 2 and 4 . This may be also interpreted, in more common terms, as a flipping of one (or several) local cluster (s). When the temperature is raised, the defects tend to become fuzzy (rough) and it becomes increasingly difficult to know precisely how the frustration network is connected by the defects; this means that it becomes increasingly difficult to distinguish which ground state one started from. The spin glass transition is reached when the defects are so fuzzy that this distinction becomes impossible. The simplest guess is that this transition state is reached when the defect width diverges, that is at the roughening transition.

Let us call $\delta(T)$ the defect width, for a given system, at temperature $T$. By definition, at the roughening transition, $\delta\left(T_{\mathrm{R}}\right)=\infty$. The conjecture that the spin glass transition $T_{\text {S.G. }}$ is the continuation of the roughening transition, when $T_{\mathrm{R}}>T_{\mathrm{c}}$, implies that

$$
\delta\left(T_{\text {S.G. }}\right)=\infty .
$$

This of course requires proof. It might be conceived that the spin glass transition is reached when the width $\delta$ reaches some finite value $\delta_{\mathrm{c}}$, that is before it actually diverges (one might even conjecture that both cases exist and that a distinction between secondorder and first-order spin glass transitions is thereby possible).
It is essential to realize that the disorder we consider is of the frustration type and that a percolation-type disorder (e.g. cutting bonds at random) does not induce zero-point defects. In the case of percolation, we expect that $T_{\mathrm{R}}$ vanishes at the same critical concentration $x_{\mathrm{c}}$ as $T_{\mathrm{c}}$, no spin-glass phase occurring beyond $x_{\mathbf{c}}$.

We have been led to these views by our study of the transition that occurs at zero temperature when $x$ (the disorder) is increased [5]. We came to the conclusion that the most sensitive quantities to detect the transition were the energy and width of an extra defect created by taking one ground state configuration and then flipping all the spins on one side of the sample. This defect energy vanishes at the transition point (it is generally considered [6], and probably correct, that this energy, for an Ising system, can be written as $(\xi)^{-(d-1)}$, where $\xi$ is the correlation length which diverges at the Curie transition). However, it was not clear for us whether the defect width behaved as $\xi$ or not; besides, we were looking for a criterion to determine whether, on leaving the ferromagnetic phase, we were entering a paramagnetic phase or a spin glass phase. And that is how we came to think that these two questions might be related.

In the language of defects, the two transition lines, on the upper part of figure 1, would then be interpreted as follows : the Curie transition line is where the defect free energy (surface tension) vanishes, the roughening transition line is where the defect width diverges, or otherwise stated, where the energy of a linear step (on a wall defect) vanishes [7]. Whereas for pure (unfrustrated) systems, the defect notion may appear subordinate to the notions which describe the order (order parameter, ...), it seems that, for those phase transitions (spin glass like) which are characteristic of impure (frustrated) systems, the defect notion becomes primordial.

If true, the connection between the roughening transition and the spin glass transition would shed new light on the spin glasses (and presumably also, on other disordered states, such as glasses themselves). Important time-dependent effects are associated with both transitions and would be better understood mutually than separately. Much knowledge has been gained recently on the defects of systems with arbitrary space dimensionalities and arbitrary order parameter symmetries and it is tempting to use all this information to make predictions for new spin glass phases. Therefore, naturally, we looked for the most direct way to test our conjecture.

However, an analysis of the literature has revealed that the study of the roughening transition in pure systems is far less advanced than the study of usual phase transitions [8]. Furthermore, external field effects are just beginning to be studied (see, for instance, the roughening transition which seems to occur in a two-dimensional Ising antiferromagnet under applied uniform field [9]). Therefore, a lot of 
work on the roughening transition in pure systems should be done before the analytical study of disorder effects. What can be done directly however, before analytical work, is a Monte-Carlo numerical simulation study. This constitutes a vast programme and we thought it worthwhile to present our predictions at this stage, in order to stimulate more activity in these directions.

Acknowledgments. - One of us (G. T.) wishes to thank P. C. Hohenberg for a conversation presenting the roughening transition as an interesting problem.

\section{References}

[1] A recent review on the roughening transition is given by : Leamy, H. J., Gilmer, G. H. and Jackson, K. A., in Surface Physics of Materials (Blakely ed., Academic, N. Y.), vol. I (1975) 121-188.

[2] See for instance :

Tholence, J. L. and Tournier, R., J. Physique Colloq. 35 (1974) C 4-229; and the special issue of Physica 86-88 (1977) $\mathrm{B}+\mathrm{C}$.

[3] Burton, W. K., Cabrera, N. and Frank, F. C., Philos. Trans. R. Soc. London 243 A (1951) 299;

Gallavotti, G., Commun. Math. Phys. 27 (1972) 103.

[4] Young, A. P. and Stinchcombe, R. B., J. Phys. C. 9 (1976) 4419.

[5] Toulouse, G., Commun. Phys. 2 (1977) 115;

Vannimenus, J. and Toulouse, G., J. Phys. C 10 (1977) L-2537;

KIRKPATRICK, S., to be published.
[6] Fisher, M. E., J. Vac. Sci. Technol. 10 (1973) 665.

[7] Leamy, H. J. and Gilmer, G. H., J. Cryst. Growth 24 (1974) 499 ; SWENDSEN, R. H., Phys. Rev. B 15 (1977) 5421.

[8] Recent attempts to relate the roughening transition to usual phase transitions are described in

Chui, S. T. and Weeks, J. D., Phys. Rev. B 14 (1976) 4978 ;

Van Beiseren, H., Phys. Rev. Lett. 38 (1977) 993;

KNOPS, H. J. F., Phys. Rev. Lett. 39 (1977) 766;

MülleR-KrumbhaAR, H., in Crystal Growth and Materials (E. Kaldis and H. J. Scheel eds., North-Holland) vol. 2 (1977) 115.

[9] Müller-Hartmann, E. and Zittartz, J., Z. Phys. B 27 (1977) 261. 